\title{
Kappa and lambda light chain mRNA in situ hybridization compared to flow cytometry and immunohistochemistry in B cell lymphomas
}

Lisa M Rimsza ${ }^{*}$, William A Day², Sarah McGinn ${ }^{1}$, Anne Pedata², Yasodha Natkunam³ ${ }^{3}$ Roger Warnke ${ }^{3}$, James R Cook ${ }^{4}$, Teresa Marafioti ${ }^{5}$ and Thomas M Grogan ${ }^{2}$

\begin{abstract}
Background: Detection of B cell clonality is useful for assisting in the diagnosis of B cell lymphomas. Clonality assessment can be accomplished through evaluation of KAPPA and LAMBDA light chain expression. Currently, only slide based methods are available for the majority of patient biopsies and do not detect light chain protein or mRNA in many B-cell lymphomas. Herein we evaluated a new method, known as colorimetric in situ hybridization (CISH), with improved sensitivity and multiplexing capacity, for its usefulness in clonality detection in mature B cell malignancies.

Methods: The KAPPA and LAMBDA ISH was performed on a Ventana Benchmark XT utilizing two color chromogenetic detection. The probes comprised 2 haptenated riboprobes each approximately 500 base pairs long directed against the conserved regions of either KAPPA or LAMBDA mRNA. The dual colors consisted of silver deposition (black) for KAPPA light chain and a novel (pink) chromogen for LAMBDA light chain. Following optimization, CISH allowed visualization of mRNA in benign B cells in reactive tissues including germinal center, mantle zone, and post-germinal center cells. We then identified 79 cases of B cell lymphoma with formalin-fixed paraffin-embedded (FFPE) biopsies including: follicular (36 cases), mantle cell (6 cases), marginal zone (12 cases), lymphoplasmacytic (6 cases), small lymphocytic (4 cases), and diffuse large B cell (15 cases), which were selected on the basis of either prior flow cytometry or immunohistochemistry (IHC) results to serve as the predicate, "gold standard," comparator.
\end{abstract}

Results: 39/79 (49.4\%) cases were classified as KAPPA and 29/79 (36.7\%) as LAMBDA light chain restricted; while 9/79 (11.3\%) cases were classified as indeterminate. Of the 70 cases with KAPPA or LAMBDA light chain restricted CISH, $69 / 70$ (98.6\%) were concordant with the reference method, while 1/70 (1.4\%) was discordant.

Conclusions: Optimized CISH detected lower levels of mRNA than can be visualized with current slide based methods, making clonality assessment in FFPE biopsies possible for mature B cell neoplasms. In this preliminary study, CISH was highly accurate compared to flow cytometry or IHC. CISH offers the possibility of wider applicability of light chain ISH and is likely to become a useful diagnostic tool.

Virtual Slides: The virtual slide(s) for this article can be found here: http://www.diagnosticpathology.diagnomx.eu/ vs/1430491067123856

Keywords: Clonality, B cell lymphoma, Kappa, Lambda, In situ hybridization, Light chains

\footnotetext{
*Correspondence: Irimsza@email.arizona.edu

'Department of Pathology, University of Arizona, Tucson, AZ, USA

Full list of author information is available at the end of the article
} 


\section{Background}

Detection of B cell clonality has long been a useful tool for identification of monoclonal B cell populations, which are potentially malignant, from polyclonal B cell populations that result during the normal immune response [1]. The copy number range of light chain mRNA and protein expressed by B cells is variable and depends on stage of differentiation. While naive and memory cells may have only 10-100 mRNA copies per cell, plasma cells may have approximately 100,000 mRNA copies per cell [1]. Therefore detection methods need to have a wide dynamic range to be suitable for application in different types of $B$ cell malignancies.

Detection of B cell monoclonality in diagnostic pathology clinical laboratories can follow one of 4 basic methods. If fresh tissue is available, then disassociation of the lymphoid cells followed by immunofluoresence staining and flow cytometric analysis is common. This technique gives a level of sensitivity suitable for detecting the level of kappa or lambda protein found on the surface of all stages of B cell development [2]. If a permeabilization step is used in cell preparation prior to staining, then cytoplasmic light chain protein can be detected in plasma cells and lymphoplasmacytic cells [3]. While a highly successful method, the need for fresh unfixed tissue excludes many cases from analysis. If lymphoma is not suspected ahead of time or only a limited amount of biopsy material is obtained, then often only fixed tissues are available. In this common situation, immunohistochemical (IHC) detection methods of kappa and lambda light chain protein in formalin fixed, paraffin embedded tissues (FFPET) are widely used. However, these are somewhat limited by high background due to the presence of normal physiologic interstitial Ig and the low abundance of $K A P P A$ and $L A M B D A$ mRNA and protein on normal and malignant germinal center and pre- germinal center B cells [4]. Assessment of mRNA avoids the issue of interstitial Ig signal since mRNA is short-lived and intra-cytoplasmic. In situ hybridization (ISH) for mRNA is therefore used in many laboratories. However, current methods allow visualization of mRNA levels of B cells only in the later stages of differentiation and so do not have the dynamic range of flow cytometry (see Figure 1) [5,6] Finally, molecular methods aimed at amplification of $K A P P A$ and $L A M B D A$ light chain genes and examination for a predominant rearrangement motif are also used. These techniques usually performed in molecular laboratories, require longer time as nucleic acid extraction and amplification are needed and do not retain morphologic context i.e. exact visualization of the cell of interest [7]. Therefore, despite a variety of current methods, there are still many cases where assessment of B cell monoclonality is technically challenging, posing a limit to the diagnostic process.

In diagnostic pathology, ISH on histologic sections commonly starts with haptenated probes (labeled with either DNP (2,4-dinitorphenol) or DIG (digoxigenin), followed by an anti-hapten antibody, and an indirect biotin streptavidin system with either alkaline phosphatase or silver deposition for visualization [8;9]. Clinical examples common in diagnostic hematopathology include detection of $K A P P A$ and $L A M B D A$ mRNA, as discussed above, as well as Epstein Barr Virus Encoded RNAs (EBER). New probe design approaches eliminating any repeat segments, as well as the development of novel haptens and chromogens have opened the possibility for more specific staining with greater sensitivity and the detection of multiple probes per slide $[8,9]$.

Given the advantages of ISH (suitability for fixed tissues, absence of background) as well as technological developments in the field, the current study sought to develop a new methodology known as dual color in situ hybridization $(\mathrm{CISH})$ and to compare its performance in

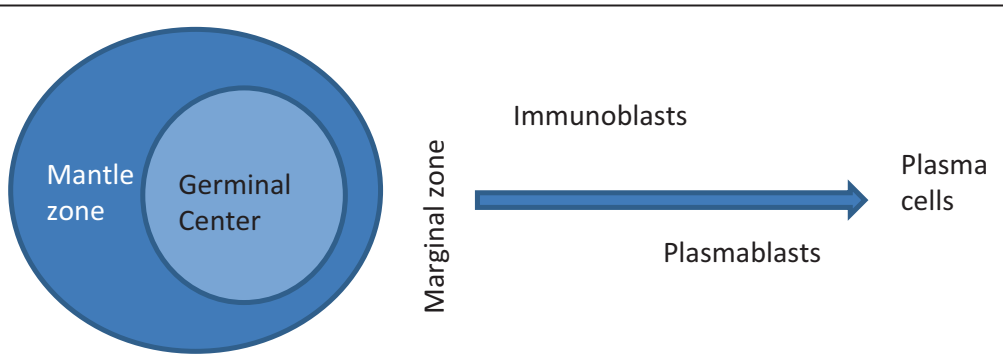

(Current slide methods detect in this range)

Figure 1 lg mRNA levels increase with B cell differentiation. As B lymphocytes pass through stages of maturation from precursor B cells to naïve $B$ cells to germinal center cells to post-germinal center cells then to plasma cells, the level of mRNA encoding immunoglobulin increases. Current slide-based methods are generally able to detect the mRNA levels found in the later stages of differentiation (generally in the postgerminal center stages). 
assessing B cell clonality to more commonly employed methods in a series of non-Hodgkin B cell lymphomas. Herein, we describe for the first time this new method and report initial promising results for future clinical application.

\section{Methods}

Tissues

Benign tissues included 2 tonsils removed for tonsillitis and 1 with reactive paracortical hyperplasia. B cell nonHodgkin lymphomas (B-NHL) cases were assembled as follows: Stanford University submitted 19 cases with flow cytometry results, Cleveland Clinic submitted 18 cases with flow cytometry results, the University of Arizona submitted 27 cases with flow cytometry results, and the University College of London submitted 15 cases with IHC results. The case criteria included a diagnosis of mature B-cell non-Hodgkin lymphoma, remaining tumor in the paraffin block, and documented monotypic light chain pattern by flow or IHC. Each site performed flow cytometry according to their own clinical laboratory standards and so was not standardized across the case series. Decalcified samples from bone marrow were not included since the method is not yet optimized for that setting. This project was conducted under a human subjects research ethical approval from the University of Arizona Institutional Review Board (protocol \# 0500000226) according to the Declaration of Helsinki.

\section{Probe synthesis and formulation}

Novel KAPPA and LAMBDA anti-sense probes, complementary to mRNA encoding each light chain constant region, were chemically labeled with different haptens using Mirus linker arms as directed by the manufacturer (Mirus Bio LLC, Madison, WI). Twenty-five nanograms of each probe was suspended in one $\mathrm{mL}$ of Ribohybe ${ }^{\text {mm }}$ (cat. 760-104, Ventana Medical Systems) solution and placed into a dispenser.

\section{RNA in situ hybridization and tyramide-chromogen detection}

All steps were executed using a VENTANA Benchmark XT automated staining instrument (Ventana Medical Systems). Formalin-fixed, paraffin-embedded tissue samples mounted on Superfrost slides were de-paraffined and antigen retrieved using $\mathrm{CC} 1$ reagent and protease 3 (cat. 760-2020, Ventana Medical Systems). Following retrieval, cocktailed hapten-labeled KAPPA and LAMBDA experimental anti-sense strand probes were dispensed onto a slide, denatured at $80^{\circ} \mathrm{C}$ for $8 \mathrm{~min}$, and hybridized at $65^{\circ} \mathrm{C}$ for $6 \mathrm{hrs}$. Following hybridization, slides were washed 3 times using $0.1 \times \mathrm{SSC}$ at $75^{\circ} \mathrm{C}$ for $8 \mathrm{~min}$. to remove non-specifically hybridized probe. Brightfield detection of each bound probe required a two-tiered sequential amplification strategy as follows. Endogenous tissue peroxidase activity was inactivated using $\mathrm{PO}$ inhibitor (cat. 760-4143, Ventana Medical Systems). The KAPPA probe was detected using an anti-KAPPA probe hapten monoclonal antibody conjugated to horseradish peroxidase/HRP to catalyze hapten amplification using a tyramidehapten conjugate and TSA- $\mathrm{H}_{2} \mathrm{O}_{2}$ reagent (cat. 760-4141, Ventana Medical Systems). The procedure was repeated to detect the labeled $L A M B D A$ probe using tyramidemediated amplification of the second hapten. Control studies demonstrated that three successive applications of $\mathrm{PO}$ inhibitor were required prior to $L A M B D A$ signal amplification to inactivate the HRP-conjugates used to amplify the KAPPA probe signal; omission of the inactivation step resulted in co-localization of signals and non-specific mRNA signals. Chromogenic detection of the $L A M B D A$ then KAPPA associated haptens was accomplished sequentially using a similar amplification strategy which included in order three applications of PO inhibitor, application of a cognate anti-hapten monoclonal antibody and application of a tyramide-sulforhodamine $\mathrm{B}$ chromogen conjugate (for $L A M B D A$ mRNA) before using a similar strategy to deposit the silver chromogen (for KAPPA mRNA) (See Figure 2). Tissue nuclei were stained using Hematoxylin; slides were then dehydrated using gradient alcohols and coverslipped. Cognate cocktailed sense strand probes were used as negative controls to determine background resulting from non-specific probe interactions.

\section{Slide interpretation}

The slides were interpreted by 3 pathologists (SM, TG, LR) without knowledge of the flow cytometry or IHC results. Each pathologist initially read all slides independently without access to each other's interpretations. Results between pathologists were then compared, followed by discrepancy resolution by joint microscopic review to achieve consensus. After a consensus interpretation was achieved, the CISH results were unblinded and compared to the flow cytometry and IHC results.

Dual color CISH for kappa/lambda mRNA is a new technology with results not before visualized. Positive staining was therefore carefully defined as demonstrating a partial to full ring of punctuate cytoplasmic staining. The signals were small and delicate similar in size to iron particles identified in erythroid precursors using an Prussian Blue stain on bone marrow aspirate smears. In cells at the late stage of B cell differentiation such as plasma or plasmacytic cells, the staining was sometimes heavy enough to obscure the entire cell. Nucleolar staining was considered non-specific. Indeterminate staining was assigned when there was a lack of or minimal staining on the malignant $B$ cells (no rings or partial rings) and the plasma cells in the sample were 


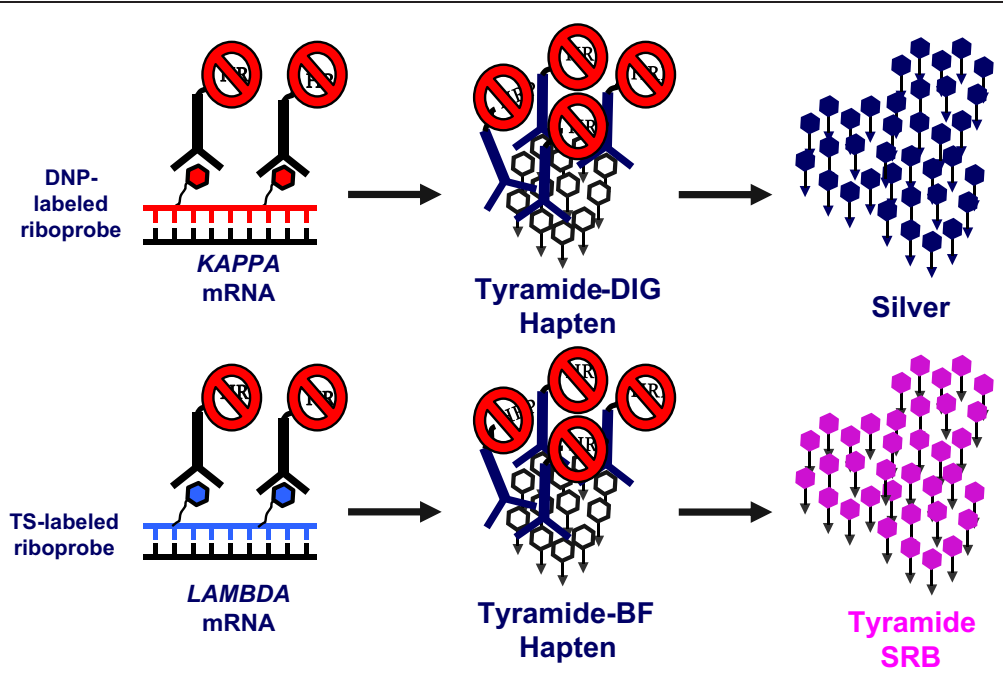

Figure 2 2-Color RNA Detection Methodology. A cocktail of antisense hapten-labeled riboprobes, targeting the constant region of either KAPPA or $\angle A M B D A$ mRNA, was hybridized to de-paraffinized protease treated tissue samples. Following stringency washes the haptens were sequentially detected using a cognate anti-hapten monoclonal antibody conjugated to HRP which catalyzed deposition of a tyramide-hapten conjugate. Following inactivation of the HRP conjugate, each amplified hapten was then sequentially detected using a second anti-hapten antibody-HRP conjugate which catalyzed silver or pink chromogen deposition.

positively stained or absent. Failed staining was assigned when there was no or minimal staining on malignant B cells (no rings or partial rings) and the plasma cells, which are expected to be strongly stained, were present but unstained.

\section{Results and discussion}

Reactive tissues demonstrated a polyclonal pattern of staining including both kappa expressing (black labeled cells) and lambda expressing (pink labeled cells) (Figure 3). Within the germinal centers, centroblasts, centrocytes,

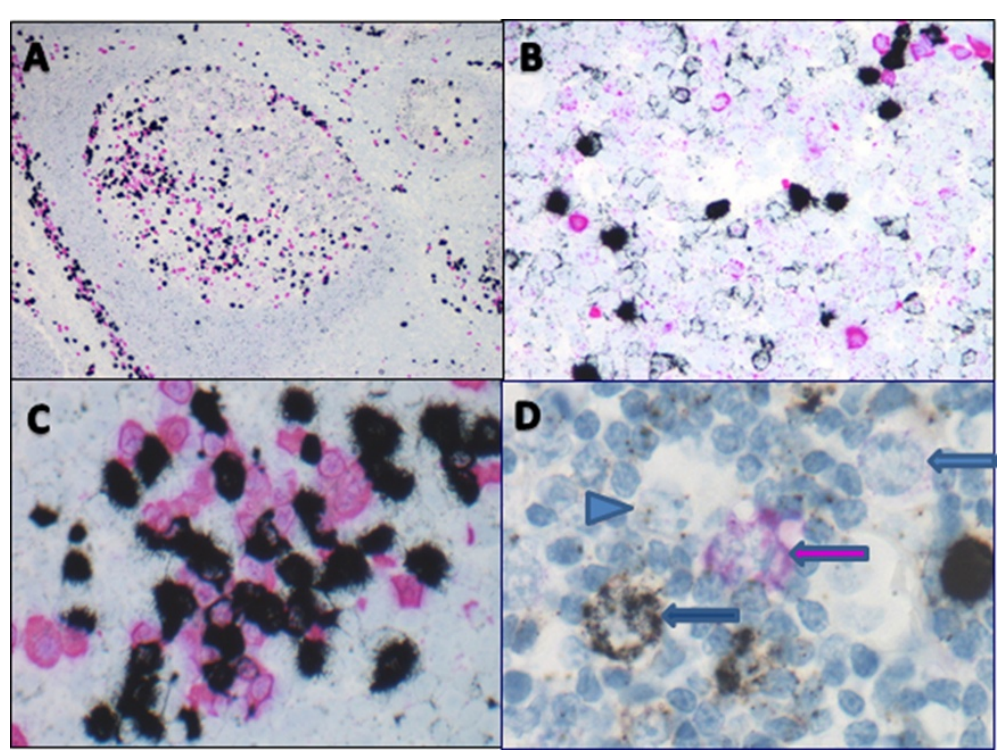

Figure 3 Staining Pattern in Reactive Lymphoid Tissues. A: Low power view of tonsil showing polyclonal staining within germinal center and mantle zone, and scattered paracortical B cells as well as intra-germinal center and paracortical plasma cells (40X). B: High power view of germinal center demonstrating polyclonal centrocytes, centroblasts, and a few plasma cells (500x). C: High power view of medullary sinuses with polyclonal plasma cells (600X). D: High power view of reactive paracortical hyperplasia showing KAPPA restricted B-immunoblast (black arrow), LAMBDA restricted B-immunoblast (pink arrow), negatively staining presumed T-immunoblast (blue arrow), and negatively staining histiocyte (blue arrowhead) (1000x). 


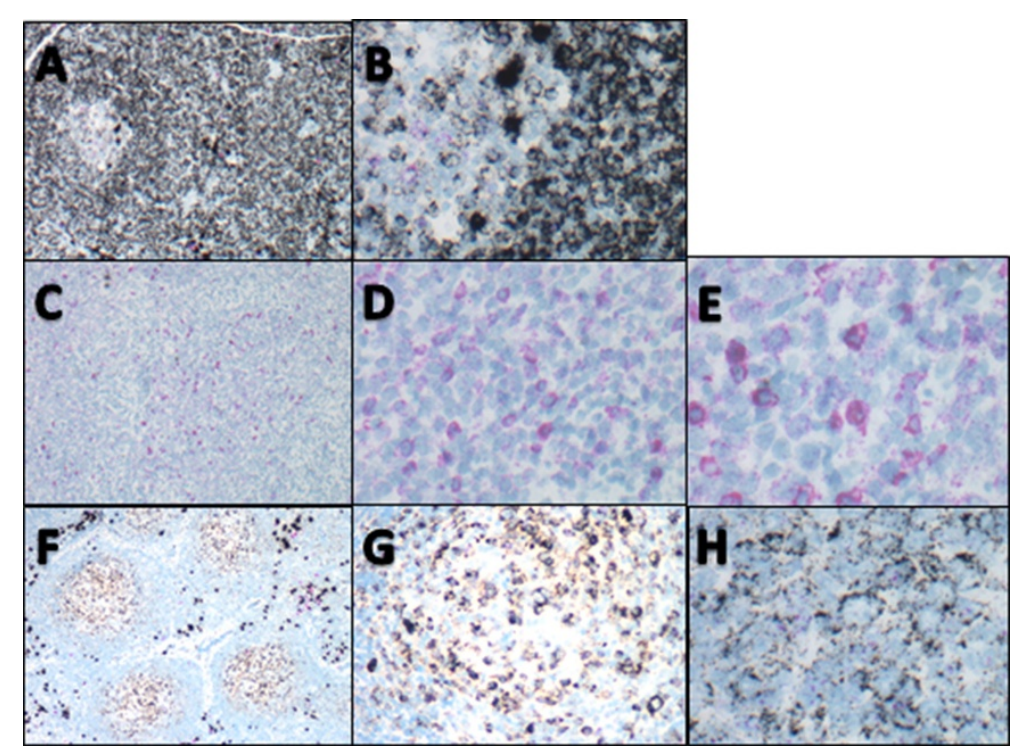

Figure 4 Light chain restriction in B cell lymphomas. A-B: CLL/SLL (A) residual germinal center on left with sheets of monoclonal KAPPA light chain restricted lymphoma cells on the right (100x); (B) higher power view of KAPPA light chain restricted cells (500X). C-E: DLBCL (C) diffuse sheets of $L A M B D A$ light chain restricted cells (100x); (D) same case showing intermediate magnification of LAMBDA light chain restricted lymphoma cells (400X); (E) same case showing higher power view with centroblastic morphology and LAMBDA light chain restriction. Rather than an inherent difference in the chromogens, but rather due to the larger cell size and lower nuclear to cytoplasmic ratios in DLBCL as Compared to $\mathrm{SLL}$ or $\mathrm{FL}$, the cellular detail is easier to appreciate in $\mathbf{E}$ than in images $\mathbf{B}$ or $\mathbf{H}(600 \times)$. F-H: Follicular lymphoma (F) low power view showing neoplastic germinal centers with KAPPA light chain restriction and cuff of polyclonal plasma cells (100X); (G) same case at intermediate power showing KAPPA light chain restricted $F L_{;}(\mathbf{H})$ same case at higher power showing KAPPA light chain restriction of both small and large neoplastic cells $(600 x)$

and plasma cells stained in a polyclonal pattern. Polyclonal staining of the mantle zone lymphocytes could also be visualized. As expected, the plasma cells in the medullary sinuses were also polyclonal. In a lymph node with reactive paracortical hyperplasia, a mixture of KAPPA and LAMBDA expressing immunoblasts were identified as were immunoblasts which were negative for both KAPPA and $L A M B D A$ as well as KAPPA and $L A M B D A$ negative immunoblasts, which were presumed to be T-cell immunoblasts.

After determining that the dual CISH assay was able to detect KAPPA and $L A M B D A$ mRNA in mature $\mathrm{B}$ cells of increasing differentiation stages as encountered in peripheral lymphoid tissues, we next collected cases of B-NHL known to arise from the various lymph node

Table 1 Total cases: concordant, discordant, indeterminate

\begin{tabular}{lll}
\hline & $\begin{array}{l}\text { Cases with minimum } \\
\text { study criteria }\end{array}$ & $\begin{array}{l}\text { Cases with interpretable } \\
\text { CISH (excluding } \\
\text { indeterminate cases) }\end{array}$ \\
\hline Total & 79 & $70 / 79(88.6 \%)$ \\
$\begin{array}{l}\text { Indeterminate } \\
\begin{array}{l}\text { Concordant with } \\
\text { flow or IHC }\end{array}\end{array}$ & $9 / 79(11.3 \%)$ & - \\
$\begin{array}{l}\text { Discordant with } \\
\text { flow or IHC }\end{array}$ & $1 / 79(1.3 \%)$ & $69 / 70(98.6 \%)$ \\
\hline
\end{tabular}

populations (germinal center-, mantle zone-, marginal zone-derived lymphomas). Benign precursor B cells found in bone marrow and lymphoblastic leukemia/ lymphomas were not included. There were 79 total cases which met all inclusion criteria representing 34 cases of follicular lymphoma (FL), 7 cases of mantle cell lymphoma (MCL), 11 cases of marginal zone lymphoma (MZL), 6 cases of lymphoplasmacytic lymphoma (LPL), 15 cases of diffuse large B cell lymphoma (DLBCL), 4 cases of chronic lymphocytic leukemia/small lymphocytic lymphoma (CLL/SLL), and 2 cases of small B-cell non-Hodgkin lymphoma, not otherwise specified. Photomicrographs of

Table 2 Concordance of evaluable cases by lymphoma diagnosis

\begin{tabular}{lcc}
\hline Diagnosis & Concordance & Discordance \\
\hline Follicular & $27 / 28(96.4 \%)$ & $1 / 28(3.6 \%)$ \\
Mantle Cell & $7 / 7(100 \%)$ & $0 / 7(0 \%)$ \\
Marginal Zone & $10 / 10(100 \%)$ & $0 / 11(0 \%)$ \\
Lymphoplasmacytic & $6 / 6(100 \%)$ & $0 / 6(0 \%)$ \\
Diffuse Large B-cell & $13 / 13(100 \%)$ & $0 / 13(0 \%)$ \\
SLL/CLL & $4 / 4(100 \%)$ & $0 / 4(0 \%)$ \\
Small cell B-NHL, NOS & $2 / 2(100 \%)$ & $0 / 2(0 \%)$ \\
Total & $69 / 70(98.6 \%)$ & $1 / 70(1.4 \%)$ \\
\hline
\end{tabular}


Table 3 Indeterminate vs evaluable by lymphoma diagnosis

\begin{tabular}{llcc}
\hline Diagnosis & Cases & Indeterminate & Evaluable \\
\hline Follicular & 34 & $6 / 34(17.6 \%)$ & $28 / 34(82.4 \%)$ \\
Mantle cell & 7 & $0 / 7(0 \%)$ & $7 / 7(100 \%)$ \\
Marginal Zone & 11 & $1 / 11(9 \%)$ & $10 / 11(91 \%)$ \\
Lymphoplasmacytic & 6 & $0 / 6(0 \%)$ & $6 / 6(100 \%)$ \\
Diffuse Large B Cell & 15 & $2 / 15(13.3 \%)$ & $13 / 15(86.7 \%)$ \\
CLL/SLL & 4 & $0 / 4(0 \%)$ & $4 / 4(100 \%)$ \\
Small B Cell NHL, NOS & 2 & $0 / 2(0 \%)$ & $2 / 2(100 \%)$ \\
Total & 79 & $9 / 79(11.3 \%)$ & $70 / 79(88.6 \%)$ \\
\hline
\end{tabular}

representative cases are shown in Figure 4. As seen in Table 1, 70/79 cases (88.6\%) were found to have either KAPPA $(39 / 79,49.4 \%)$ or LAMBDA (29/79, 36.7\%) light chain restriction in the presence of appropriate residual plasma cell staining. The remaining cases were classified as indeterminate $(9 / 79,11.3 \%)$ using the criteria described in Methods. Of these 9 indeterminate cases, 5 lacked staining in B cells with positive plasma cell staining and 4 lacked staining in B cells without discernible plasma cells in the section (to serve as tissue positive control). Therefore 75/79 (95\%) of cases were considered to have sufficient mRNA integrity to be reliably interpreted. Of the 70 cases with interpretable CISH, 69/70 (98.6\%) were concordant with the reference method (either flow cytometry or IHC), while $1 / 70(1.4 \%)$ was discordant (shown by diagnostic category in Table 2). The latter case was a follicular lymphoma that showed KAPPA restriction by CISH and $\angle A M B D A$ by IHC. Further examination of the discrepant cases demonstrated that 6/34 FL (17.6\%), 1/11 MZL (9\%), and 2/15 DLBCL (13.3\%) were deemed indeterminate by
CISH but were found to be evaluable by flow cytometry or IHC. These cases are detailed in Table 3.

Interestingly, 2 cases which were negative for surface Ig by flow cytometry were submitted to the study. Surface Ig negativity is well recognized and considered a sign of monoclonality in the workup of B-NHL, and has been reported in a variety of $\mathrm{B}$ cell lymphomas including follicular lymphoma [10-12]. In this study, 2 FL were surface Ig negative by flow cytometry. Both of these cases had "indeterminate" CISH results. Of these, 1 case contained positively stained polyclonal plasma cells (a true SIg negative case by all methods), while the other case did not contain any discernable plasma cells to indicate that staining was or was not successful. This latter case could therefore be considered either a true negative (non-Ig expressing lymphoma) or perhaps a case with poorly preserved mRNA.

A non-specific nucleolar staining was seen with the $L A M B D A$ probe in 14 cases including all categories of lymphoma (a representative case is shown in Figure 5). Among the 14 cases, 6 showed KAPPA restriction, 5 $L A M B D A$, and 3 were classified as "indeterminate" by $\mathrm{CISH}$. Because of the preservation of tissue morphology, this nucleolar staining was easily distinguished from the specific cytoplasmic pattern of KAPPA or LAMBDA mRNA staining.

When considering the diagnosis of lymphoma in patient biopsies, the monoclonality or polyclonality of the B cell populations is a critical component of the decision making process. While many methods can be used, the most common (flow cytometry) requires fresh tissue, or does not take into account morphologic context (PCR), or is insufficiently sensitive for the majority of $\mathrm{B}$ cell NHL (current IHC and ISH methods) [5,6]. In this

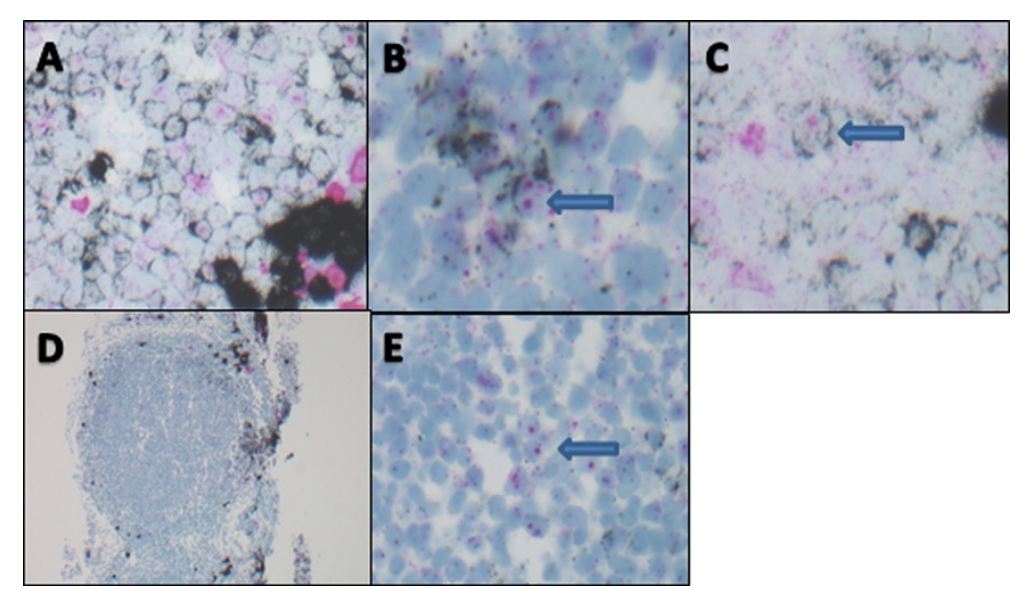

Figure 5 Non-specific nucleolar staining with lambda probe. A-C: Germinal center in a reactive tonsil. (A) polyclonal mixture of KAPPA and LAMBDA positive cells $(400 \times)$; (B\&C): arrows point out KAPPA expressing cells as evidenced by black ring of cytoplasmic staining with non-specific pink nucleolar staining (600X). D-E: Follicular lymphoma. (D) low power view of FL case which was indeterminate for KAPPA and lambda due to expressing neither light chain (40X); (E): high power view of same case demonstrating lack of cytoplasmic staining pattern for either KAPPA or LAMBDA light chain expression with non-specific pink nucleolar staining (600x). 
study, we describe the feasibility and application of dual-color CISH technology for the evaluation of clonality of mature B cell populations in tissue sections. We demonstrate that with new, repeat-free probes and sensitive detection systems taking advantage of tyramideinduced deposition of chromogens, it is possible to detect not only KAPPA and $\angle A M B D A$ mRNA levels typical of plasma cells and other post-germinal center $B$ cells but even the very low level mRNA found in presumed naïve $B$ cells in the mantle zones and germinal center B cells. This level of detection is then applicable to the lymphomas derived from most stages of $\mathrm{B}$ cell differentiation. The ability to automate the staining and then evaluate the results using light microscopy for slide evaluation holds the potential to easily integrate the CISH technique into routine diagnostic practice.

\section{Conclusions}

In this study we describe a preliminary accuracy of $98 \%$ as compared to reference methods including flow cytometry and immunohistochemistry. There was only 1 case called KAPPA by CISH and $L A M B D A$ by IHC, which is difficult to explain biologically. However, the explanation could be as simple as a technical or transcriptional error in the original report. Nevertheless, biological complexity does exist in the interpretation of the lambda CISH results due to the presence of non-specific nucleolar staining with the $L A M B D A$ probe seen in several cases. Initially, this staining seemed to suggest conflicting results, particularly in the cases which had clear KAPPA mRNA cytoplasmic black colored staining while having apparent " $L A M B D A$ " pink staining in the nucleoli of the same cells. However, a review of the literature revealed that this staining pattern may result from cross hybridization with the Ig lambda-like polypeptide 5 (IGLL5) gene on chromosome 22, which is located within the immunoglobulin lambda locus but does not require somatic rearrangement for expression, meaning that it can be expressed in non-Ig expressing, non-B, cells [13]. As recently demonstrated by Tubbs et al. cross-hybridization with the LAMBDA CISH probe occurs because while the first exon of IGLL5 gene is unrelated to immunoglobulin variable genes, the second and third exons use the same loci as the Ig-lambda joining 1 and the Ig-lambda constant 1 gene segments [13,14]. Recognition of this non-specific pattern allowed interpretation of cases with pink nucleolar staining as well as black cytoplasmic staining as KAPPA restricted. In contrast, those with pink nucleolar staining without cytoplasmic staining were scored as "indeterminate". In at least 2 cases, "indeterminate" is the most accurate interpretation since by flow cytometry these were both considered surface Ig-negative cases.

Dual-color CISH allowed for detection of both KAPPA and $L A M B D A$ mRNA on a single slide under a light microscope, making interpretation of B-cell clonality in tissue sections reliable and highly optimized in the context of tissue architecture and cellular compartments. This application should be of particular interest to pathologists engaged in the field of lymphoma diagnostics. The availability of additional novel haptens and chromogens, which can be paired with highly specific probes, allows for the possibility of additional multi-color combinations and innumerable future applications.

\section{Competing interests}

Bill Day, Anne Pedata, and Tom Grogan are employees of Ventana Medical Systems, which supplied the probes and performed the staining for the project.

\section{Authors' contributions}

LR designed the study, interpreted results, and wrote the manuscript. WD designed the reagents, developed the techniques, and supervised experiments. SM identified cases, interpreted results, and wrote portions of the manuscript. AP designed the reagents, developed the techniques, and performed experiments. YN assisted in study design, supplied case materials, evaluated staining. RW conceived of the study, supplied case materials. JRC assisted in study design, supplied case materials. TM assisted in study design, supplied case materials. TMG conceived of the study, interpreted results, assembled data. All authors read and approved the final manuscript.

\section{Author details}

${ }^{1}$ Department of Pathology, University of Arizona, Tucson, AZ, USA. ²Ventana Medical Systems, Inc., Tucson, Arizona, USA. ${ }^{3}$ Department of Pathology, Stanford University College of Medicine, Stanford, CA, USA. ${ }^{4}$ Robert J. Tomsich Pathology and Laboratory Medicine Institute, Cleveland Clinic, Cleveland, OH, USA. ${ }^{5}$ Department of Pathology, University College Hospital London, London, UK

Received: 18 March 2014 Accepted: 27 June 2014 Published: 21 July 2014

\section{References}

1. Segal GH, Shick HE, Tubbs RR, Fishleder AJ, Stoler MH: In situ hybridization analysis of lymphoproliferative disorders. Assessment of clonality by immunoglobulin light-chain messenger RNA expression. Diagn Mol Pathol 1994, 3:170-177.

2. de Tute RM: Flow cytometry and its use in the diagnosis and management of mature lymphoid malignancies. Histopathology 2011, 58:90-105.

3. Tembhare PR, Yuan CM, Venzon D, Braylan R, Korde N, Manasanch E, Zuchlinsky D, Calvo K, Kurlander R, Bhutani M, Tageja N, Maric I, Mulquin M, Roschewski M, Kwok M, Liewehr D, Landgren O, Stetler-Stevenson M: Flow cytometric differentiation of abnormal and normal plasma cells in the bone marrow in patients with multiple myeloma and its precursor diseases. Leuk Res 2014, 38:371-376.

4. Beck RC, Tubbs RR, Hussein M, Pettay J, Hsi ED: Automated colorimetric in situ hybridization (CISH) detection of immunoglobulin (lg) light chain mRNA expression in plasma cell (PC) dyscrasias and non-Hodgkin lymphoma. Diagn Mol Pathol 2003, 12:14-20.

5. Magro C, Crowson AN, Porcu P, Nuovo JG: Automated kappa and lambda light chain mRNA expression for the assessment of B-cell clonality in cutaneous B-cell infiltrates: its utility and diagnostic application. J Cutan Pathol 2003, 30:504-511.

6. Rimsza L, Vela E, Richter L, Bellamy W, Grogan T: Assessment of kappa and lambda light chain mRNA in paraffin embedded bone marrow core biopsies and lymph nodes using rapid automated colorimetric in situ hybridization. Lab Invest 1997, 76:775.

7. van Dongen JJ, Langerak AW, Bruggemann M, Evans PA, Hummel M, Lavender FL, Delabesse E, Davi F, Schuuring E, García-Sanz R, van Krieken JH, Droese J, González D, Bastard C, White HE, Spaargaren M, González M, Parreira A, Smith JL, Morgan GJ, Kneba M, Macintyre EA: Design and standardization of PCR primers and protocols for detection of clonal immunoglobulin and T-cell receptor gene recombinations in suspect 
lymphoproliferations: report of the BIOMED-2 Concerted Action BMH4-CT98-3936. Leukemia 2003, 17:2257-2317.

8. Nitta H, Hauss-Wegrzyniak B, Lehrkamp M, Murillo AE, Gaire F, Farrell M, Walk E, Penault-Llorca F, Kurosumi M, Dietel M, Wang L, Loftus M, Pettay J, Tubbs RR, Grogan TM: Development of automated brightfield double in situ hybridization (BDISH) application for HER2 gene and chromosome 17 centromere (CEN 17) for breast carcinomas and an assay performance comparison to manual dual color HER2 fluorescence in situ hybridization (FISH). Diagn Pathol 2008, 3:41.

9. Nitta H, Zhang W, Kelly BD, Miller M, Pestic-Dragovich L, Bieniarz C, Vasicek TJ, Marafioti T, Rimsza L, Grogan TM: Automated brightfield break-apart in situ hybridization (ba-ISH) application: ALK and MALT1 genes as models. Methods 2010, 52:352-358.

10. de Martini RM, Turner RR, Boone DC, Lukes RJ, Parker JW: Lymphocyte immunophenotyping of B-cell lymphomas: a flow cytometric analysis of neoplastic and nonneoplastic cells in 271 cases. Clin Immunol Immunopathol 1988, 49:365-379.

11. Gregg EO, Al-Saffar N, Jones DB, Wright DH, Stevenson FK, Smith JL: Immunoglobulin negative follicle centre cell lymphoma. $\mathrm{Br} J$ Cancer 1984, 50:735-744.

12. Ngan B, Warnke A, Cleary ML: Variability of immunoglobulin expression in follicular lymphoma. An immunohistologic and molecular genetic study. Am J Pathol 1989, 135:1139-1144.

13. Tubbs RR, Wang H, Wang Z, Minca EC, Portier BP, Gruver AM, Lanigan C, Luo Y, Cook JR, Ma XJ: Ultrasensitive RNA in situ hybridization for detection of restricted clonal expression of low-abundance immunoglobulin light chain mRNA in B-cell lymphoproliferative disorders. Am J Clin Pathol 2013, 140:736-746.

14. Bossy D, Milili M, Zucman J, Thomas G, Fougereau M, Schiff C: Organization and expression of the lambda-like genes that contribute to the mu-psi light chain complex in human pre-B cells. Int Immunol 1991, 3:1081-1090.

doi:10.1186/1746-1596-9-144

Cite this article as: Rimsza et al:: Kappa and lambda light chain mRNA in situ hybridization compared to flow cytometry and immunohistochemistry in B cell lymphomas. Diagnostic Pathology 2014 9:144.

\section{Submit your next manuscript to BioMed Central and take full advantage of:}

- Convenient online submission

- Thorough peer review

- No space constraints or color figure charges

- Immediate publication on acceptance

- Inclusion in PubMed, CAS, Scopus and Google Scholar

- Research which is freely available for redistribution 